\title{
Regularizing Aperiodic Cycles of Resonant Radiation in Filament Light Bullets
}

\author{
Carsten Brée \\ Weierstrass Institute for Applied Analysis and Stochastics, Mohrenstraße 39, 10117 Berlin, Germany \\ Ihar Babushkin, Uwe Morgner, and Ayhan Demircan \\ Institute for Quantum Optics, Leibniz, Universität Hannover, Welfengarten 1, 30167 Hannover, Germany
}

(Received 11 October 2016; published 17 April 2017)

\begin{abstract}
We demonstrate an up to now unrecognized and very effective mechanism which prevents filament collapse and allows persistent self-guiding propagation retaining a large portion of the optical energy on axis over unexpected long distances. The key ingredient is the possibility of continuously leaking energy into the normal dispersion regime via the emission of resonant radiation. The frequency of the radiation is determined by the dispersion dynamically modified by photogenerated plasma, thus allowing us to excite new frequencies in spectral ranges which are otherwise difficult to access.
\end{abstract}

DOI: 10.1103/PhysRevLett.118.163901

The filamentation of intense femtosecond laser pulses in transparent media represents, due to the interplay of basic linear and nonlinear effects resulting from classical and quantum mechanical approaches, a physical system of interest for fundamental science as well as for a variety of applications [1-4]. It is used for the investigation of, e.g., supercontinuum [5], high harmonic [6], or terahertz generation [7], few-cycle pulse generation [8], or complex nonlinear dynamics as self-organization [9]. Analogies to other areas of physics as artificial event horizons [10] or the ubiquitous rogue wave formation [11-13] have been shown, providing a useful test for the investigation Hawking radiation [14] or the predictability of extreme events [15].

The classical picture of the filamentation process, which was until recently thought to be well established for nearly half a century, is based on the equilibrium between the spatial collapse due to Kerr nonlinearity and the plasma defocusing. Recently and rather unexpectedly, this understanding has been questioned, especially in novel unusual regimes such as ultrashort pulses or long wavelengths. In particular, the availability of new midinfrared ultrashort high-energy laser sources led to new unusual phenomena. In Ref. [16], a superhigh-power filamentation regime in air has been revealed, which is quantitatively and qualitatively different from a conventional filament. The main point is that leaking energy into higher harmonics plays an important role in arresting the collapse. The fact that there are other efficient mechanisms for this besides the impact of plasma is regarded to trigger a fundamental paradigm shift in the field of extreme nonlinear optics. In Ref. [17], another new kind of significantly different filamentation process has been observed in bulk fused silica by pumping into the anomalous dispersion regime. This regime is known to exhibit much more complicated dynamics but also to include the advantage of solitary solutions with full spatiotemporal localization and stationarity, known as light bullets [18-26]. In a filament, these conditions cannot be exactly fulfilled $[1,2]$ due to a high number of inherent perturbations [27], but the observations in Ref. [17] demonstrate unexpected long light-bullet propagation and reveal also another interesting point. The propagation dynamics are accompanied by the generation of blueshifted radiation [28], a phenomenon which is observed also under different conditions [8,29-32] even for short filament lengths.

The other, rather independent, field of research which is actively developing in parallel to filament science but with almost no overlap is one-dimensional pulse propagation in waveguides. Dynamics of solitary waves, which appear in an anomalous dispersion range, are accompanied typically by a phase-matched radiation in the normal dispersion regime [33-35]. This so-called resonant radiation (RR) concept represents a central process in nonlinear waveguide optics (see Refs. [35,36], and references within) with a remarkably rich variety of applications. There is still an increasing interest on RR, as quite recently its ability to open new routes for highly coherent light sources with an unprecedented spectral range has been demonstrated [37-40]. However, the investigations are mainly restricted to the one-dimensional case. The interaction with photogenerated plasma is rather unknown but promises completely new possibilities for RR components [41].

Here we demonstrate that RR takes place in filaments in a similar way like in optical waveguides, but with an even more dramatic influence on the dynamics. (i) RR provides a new and effective mechanism acting against a beam collapse and enabling the stable and long on-axis channeling of optical energy, in strong difference to the conventional filamentation process. (ii) The conditions for the RR generation are drastically changed by the plasma contribution, shifting the excitation peaks to positions which are otherwise not possible. This opens up completely new possibilities for exploiting RR to generate high intense and coherent radiation in different spectral ranges. 
Our model [4] is based on the forward Maxwell equation of the analytical signal [42], augmented by Raman and plasma nonlinearities according to

$$
\begin{aligned}
\partial_{z} \mathcal{E}_{\omega}= & \frac{i}{2 \beta(\omega)} \Delta_{\perp} \mathcal{E}_{\omega}+i \beta(\omega) \mathcal{E}_{\omega}-\frac{\mu_{0} \omega}{2 \beta(\omega)} \hat{J}+i \frac{3 \omega^{2} \chi^{(3)}}{8 c^{2} \beta(\omega)} \\
& \times\left\{\left[(1-g)|\mathcal{E}|^{2}+g h_{R}(t) \circ|\mathcal{E}(t)|^{2}\right] \mathcal{E}(t)\right\}_{\omega>0},
\end{aligned}
$$

where $\mathcal{E}_{\omega}$ denotes the analytic signal of the electric field $E(t)$ in the frequency domain, the free electron current $\hat{J}$ is governed by the Drude model and a rate equation for the instantaneous electron density according to the widely used tunnel ionization model of Ammosov, Delone, and Krainov [43] for crystals, $g$ is the fractional contribution of the Raman delayed nonlinearity, o denotes the convolution integral, $h_{R}(t)$ is the Raman kernel, and $\beta(\omega)=\omega n_{0}(\omega) / c$ is the propagation constant related to the refractive index $n_{0}(\omega)$ of bulk fused silica [44]. For the numerical solution of Eq. (2), we use a standard dealiased pseudospectral splitstep method in combination with a 4th-order Runge-Kutta integration scheme for the higher-order nonlinearities with the implementation of an adaptive step-size control [4]. This numerical method has been shown to reproduce the filament dynamics in perfect agreement with experimental results [45]. The input pulsed beam has a center wavelength of $1900 \mathrm{~nm}$ in the anomalous dispersion regime, a Gaussian temporal profile with $t_{p}=50 \mathrm{fs}$, and a Gaussian beam waist $w_{0}=200 \mu \mathrm{m}$. The injected pulse has a peak power of 26 critical powers in fused silica, which amounts to an input pulse energy of $20 \mu \mathrm{J}$. For our chosen initial conditions, the evolution of the generated peak plasma density is shown in Fig. 1(a). We observe that the plasma density does not exceed $0.2 \rho_{c}$, where $\rho_{c}=\omega_{0}^{2} m_{e} \epsilon_{0} / q_{e}^{2}$ is the critical plasma density for which optical breakdown effects are likely to induce damage. Besides, we checked that the peak fluences do not exceed $2 \mathrm{~J} / \mathrm{cm}^{2}[46,47]$. The evolution of the onaxis temporal intensity profile is depicted in Fig. 1(b). The first observation is that the pulse can maintain a high fluence along the entire propagation distance of $3.5 \mathrm{~cm}$ [cf. Fig. 1(c)], in agreement with recent experimental and theoretical findings in Ref. [17].

Second, the global temporal and spectral dynamics bears a significant resemblance to the periodic excitation of RR [48-50] in guided wave nonlinear optics. In the case of propagation in a fiber, a higher-order soliton experiences periodic compression cycles, leading to a periodic overlap of the spectrum with a phase-matched component in the normal dispersion regime. This leads to a periodic excitation of RR. In a similar way, in our filament, the pulse maintains several temporal slices with sufficient optical power to induce aperiodic recurrent focusing or refocusing events according to the dynamic spatial replenishment model [51] (in contrast to existing periodic solutions mentioned above). As individual time slices refocus,

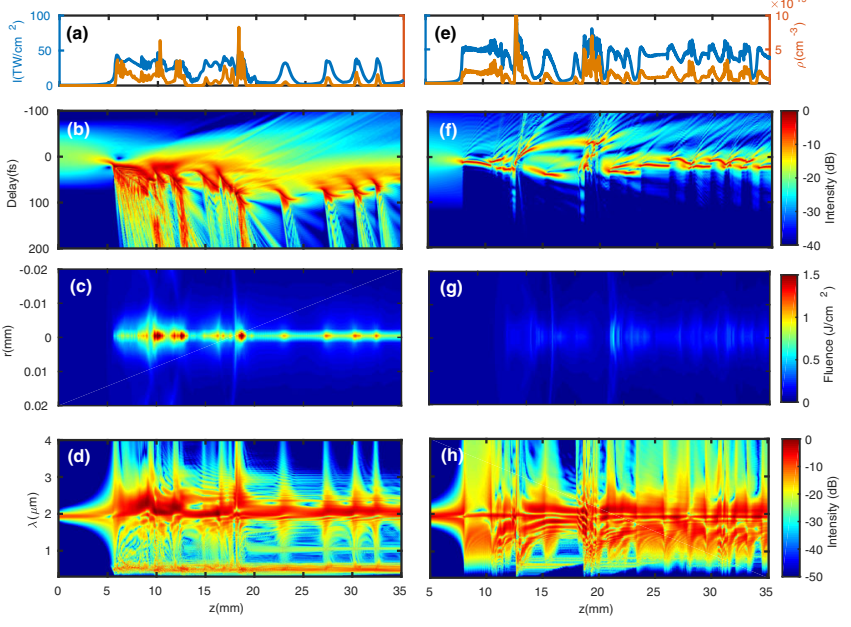

FIG. 1. (a) Peak electron density (orange curve) and peak intensity (blue curve) along optical axis $z$ for a filament seeded by a $20 \mu \mathrm{J}, 1900 \mathrm{~nm}$ pulse, simulated using the numerical model with the full dispersion profile. (b) Corresponding aperiodic evolution of the on-axis temporal intensity profile. (c) Filament fluence versus radial longitudinal filament coordinates and (d) spectral evolution along $z$ at $r=0$. Panels (e)-(h) depict the equivalent information for the same pulse, simulated using dispersion including only the $\beta_{2}$ term.

they propagate quasistationary along a distance of some millimeters and are temporally compressed and shifted towards the trailing pulse edge due to the interplay of self-steepening and anomalous dispersion [44]. Every time the corresponding spectral broadening exhibits an overlap to a phase-matched component, a pronounced emission of resonant radiation takes place [Fig. 1(d)], transferring the energy into the normally dispersive frequency domain. The corresponding resonance condition will be discussed later. In addition, upon self-focusing, the pulse envelope gets strongly modulated and splits into temporal components copropagating with the main pulse at roughly the same group velocity. This is also reminiscent of the soliton splitting dynamics induced by modulation instability (MI) [49]. The spectral evolution shown in Fig. 1(d) reveals that the emitted RR occupies multiple spectral peaks which, as $z$ grows, evolve into a quasicontinuous spectrum of RR around the wavelength range between $\lambda=600$ and $300 \mathrm{~nm}$. The amount of energy in the RR depends on its spectral overlap with the pump. We performed a number of simulations by varying the input parameters (power, wavelength, beam waist, and pulse width) and observed this behavior over wide ranges in the parameter space, demonstrating a strong robustness rather than the necessity for a delicate choice of parameters.

To demonstrate that the emission of normally dispersive RR plays an important role in regularizing the collapse dynamics, we performed additional simulations with a reduced parabolic dispersion model: $\beta(\omega)=\beta\left(\omega_{0}\right)+$ $1 / v_{g}\left(\omega-\omega_{0}\right)+1 / 2 \beta_{2}\left(\omega-\omega_{0}\right)^{2}$, where $v_{g}=1 / \beta_{1}$ is the 
group velocity and $\beta_{2}=-810 \mathrm{fs}^{2} / \mathrm{cm}$ is the groupvelocity dispersion (GVD) coefficient for silica at $\omega_{0}$, respectively. This choice completely eliminates the higher-order dispersion, and no RR can be emitted. For the same pulse energy of $20 \mu \mathrm{J}$ as in Figs. 1(a)-1(d), peak plasma densities of $50 \%$ of the critical plasma density $\rho_{c}$ are reached, which is much closer to the critical breakdown threshold than in the case of the realistic dispersion. It is necessary to mention that the employed high input powers close to the damage threshold allow a better comparison between the results with the full and reduced dispersion models, especially concerning the filament length. The corresponding evolution of the intensity, peak plasma density, on-axis temporal profile, fluence, and spectrum are shown in Figs. 1(e)-1(h). The overall observed dynamics are obviously different. Interestingly, in Fig. 1(f), defined light bullets emerge, exhibiting stable propagation along several millimeters, but abruptly disappear and reappear on axis. The strong distinctiveness of the filamentation scenario with the possibility of RR becomes evident in the evolution of the optical energy in the transverse spatial domain as shown in Figs. 1(c) and 1(g), for the case with and without a zero-dispersion wavelength (ZDW), respectively. With a ZDW, a huge part of the energy is retained on axis in a stable channel along the whole filament length [Fig. 1(c)]. The observed behavior is at first sight similar to the scenario observed in Ref. [16]. However, here neither shock formation nor third harmonics are required to allow the leakage of the radiation from the fundamental to other frequencies.

To investigate the impact of RR generation on lightbullet formation, we repeated the same approach at a low input energy $\left(5.6 \mu \mathrm{J}, 5 P_{c r}\right)$ and a center wavelength of $1800 \mathrm{~nm}$, with only a few focusing or refocusing events [Figs. 2(a) and 2(b)]. With the full dispersion profile, we observe the excitation of RR at approximately $z=4,5$, $12 \mathrm{~mm}$ [Fig. 2(a)] but with relatively long persistent propagation of the main on-axis part of the filament. The reduced, $\beta_{2}$-only model shows a completely different behavior. Here, the initial pulse splits into two pronounced light bullets propagating stably between $z=4$ and $10 \mathrm{~mm}$ [Fig. 2(c)]. Their individual trajectories appear much more stable, and the individual pulse durations are much shorter than in the full-model case. In Figs. 2(b) and 2(d), we illustrate the spatiotemporal profiles of the emergent light bullets at $z=7 \mathrm{~mm}$. Shifting the central wavelength to $2000 \mathrm{~nm}$, away from the ZDW, we expect the emission of RR to become less effective [49]. In fact, the resulting temporal evolution in Figs. 2(e) and 2(f) reveals that the filament scenario is now closer to the pure light-bullet scenario shown in Figs. 2(c) and 2(d).

To further elucidate the physical mechanisms leading to the formation of RR, we visualize the temporal dynamics of different frequency components with the cross-correlation
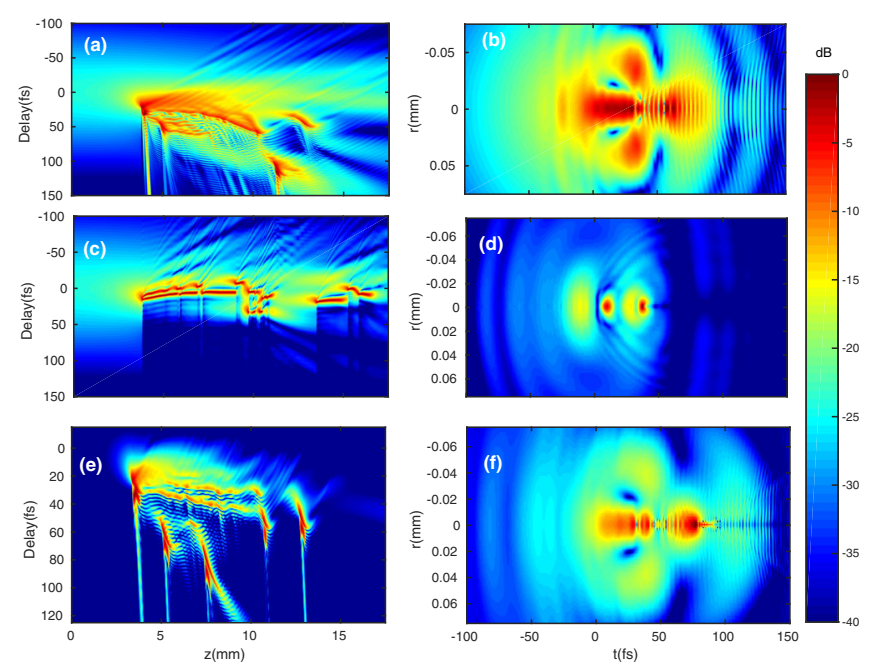

FIG. 2. Pulse dynamics for a lower peak power for different wavelengths and dispersion profiles. (a) On-axis temporal intensity profile for a $5.6 \mu \mathrm{J}$ pulse at $1800 \mathrm{~nm}$, the model with full dispersion. (b) Train of light bullets at $z=7 \mathrm{~mm}$. (c),(d) The same as (a),(b) but with reduced parabolic dispersion. (e),(f) The same as (a),(b) but for a $7 \mu \mathrm{J}$ pulse at $2000 \mathrm{~nm}$.

frequency-resolved optical gating (XFROG) technique [52] [Fig. 3(a)]. The emitted radiation is localized on several distinct branches, the dynamics of which is subject to an equation of motion governed by the inverse group velocity $k_{1}=d k(\omega) / d \omega$, according to $d t(\omega) / d z=k_{1}[\rho(z), \omega]-$ $1 / v_{R}$, where $v_{R}$ is the velocity of the comoving reference frame. Here, the dependence of $k_{1}$ on the electron density $\rho(z)$ generated at $z$ can be derived from the Drude relation $k(\omega)=\omega / c\left[n_{0}(\omega)^{2}+\omega_{p}^{2} / \omega^{2}\right]^{1 / 2}[53]$, where $\omega_{p}=$ $\sqrt{\left.\rho e^{2} / \varepsilon_{0} m_{e}\right)}$ is the plasma frequency. Figure 3(b) shows the derived $k_{1}=d k(\omega) / d \omega$ curves for different ratios of $\rho / \rho_{c}$,
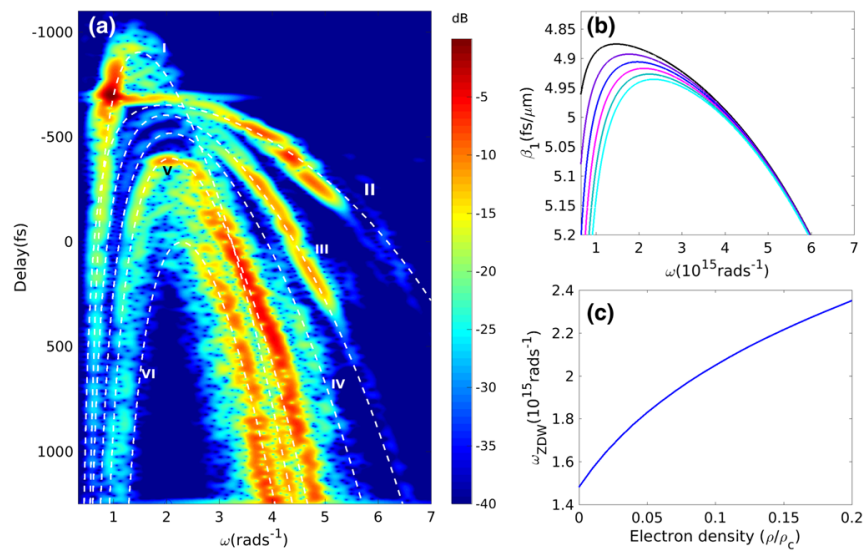

FIG. 3. (a) Spectrogram of the on-axis temporal intensity profile at $z=3 \mathrm{~cm}$ (for more details, see Supplemental Material [54]). White dashed lines: Fit according to Eq. (2) of branches I-VI. (b) Inverse group velocity for an increasing electron density. (c) ZDW frequency vs the free electron density. 
where the black curve indicates the inverse GV of fused silica for $\rho=0$. In fact, we find that higher electron densities shift the ZDW towards bluer frequencies, as also shown in Fig. 3(c). Integrating the simplified evolution equation for the branches of radiation in the XFROG trace, we obtain $t(\omega, z)=t_{0}(\omega)+\int_{z_{0}}^{z} d z k_{1}[\rho(z), \omega]-1 / v_{R}\left(z-z_{0}\right)$. By virtue of the first mean value theorem of integration, this can be simplified to

$$
t(\omega, z)=t_{0}(\omega)+\left\{\frac{1}{v_{R}}-k_{1}[\rho(\xi), \omega]\right\}\left(z-z_{0}\right)
$$

for some intermediate $\xi \in\left[z_{0}, z\right]$. In order to fit these curves to the numerically obtained XFROG trace, we assume that, immediately after emission, we observe that each branch results from a burst of resonant radiation occurring at a particular coordinate tuple $\left(t_{0}, z_{0}\right)$. By close inspection of both Fig. 1(b) and the evolution of the XFROG trace versus $z$, we find that branches I-VI correspond to six dominant radiation bursts located at $\left(z_{0}[\mathrm{~mm}], t_{0}[f s]\right)=(27.2,74)$, $(22.8,83), \quad(18.2,83.6), \quad(15.7,60.5), \quad(12.2,60.83)$, and $(9.1,75.8)$. Therefore, the only remaining free parameter in Eq. (2) is the intermediate electron density $\rho(\xi)$, and the best fit to the XFROG trace can be obtained by choosing $\rho(\xi)=$ $[0.1,0.04,0.7,0.15,0.3,0.6] \times 10^{20} \mathrm{~cm}^{-3}$ for branches I-VI, respectively. These values are close to the actual electron densities generated at the radiation bursts [cf. Fig. 1(a), brown line]. Furthermore, our analysis confirms that the dispersion of the blue spectral wings is strongly affected by plasmainduced modifications of the refractive index (see also Supplemental Material [54] for more details which present a movie sequence of the XFROG evolution).

For a further analysis of the impact of the plasma GVD on the RR, we have to consider the spatiotemporal MI which acts on the filamenting pulses. According to Ref. [44], curves of constant exponential MI gain in the $\left(k_{\perp}, \omega\right)$ plane are defined by

$$
k^{2}(\omega)-k_{\perp}^{2}-\left[k_{0}-\Delta k+\left(\omega-\omega_{0}\right) / v_{g}\right]^{2}=0,
$$

where $k_{0}$ and $k_{\perp}$ denote the wave vector at the center frequency and the transverse wave vector component, respectively, $v_{g}$ is the corresponding group velocity, and $\Delta k$ is the phase mismatch. The impact of plasma dispersion on $k(\omega)$ is modeled according to Ref. [53]. Solving Eq. (3) for $k_{\perp}=0$, the RR frequency exhibits a considerable blueshift for an increasing electron density, as shown in Fig. 4(a). To compare the predictions of Eq. (3) with the simulation results corresponding to Fig. 1, in Fig. 4(b) we show the Fourier-Hankel transform of the electric field $\mathcal{E}(r, t)$ at $z=19.2 \mathrm{~mm}$. In the anomalous dispersion regime, the impact of spatiotemporal MI is seen from the annular structures around $\omega_{0}$ and $k_{\perp}=0$. In the normal dispersion regime, the resonant radiation occupies hyperbola in the $\left(k_{\perp}, \omega\right)$ plane. The solutions of Eq. (3) for
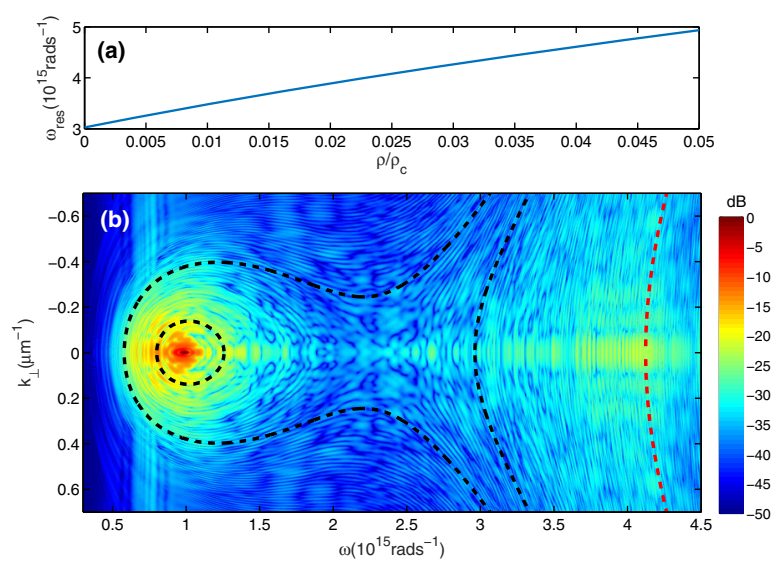

FIG. 4. (a) Frequency of RR according to Eq. (3) vs the electron density, for $\Delta k=0$ and $k_{\perp}=0$. (b) Fourier-Hankel transform of $\mathcal{E}(r, t)$ at $z=19.2 \mathrm{~mm}$. Black dashed lines: MI gain curves for $\rho=0$ and $\Delta k=20$ and $150 \mathrm{~cm}^{-1}$. Red dashed line: hyperbolic component of the curve for electron density $8.3 \times 10^{18} \mathrm{~cm}^{-3}$ and $\Delta k=20 \mathrm{~cm}^{-1}$.

vanishing electron densities are shown as black dashed lines and are in reasonable agreement with the energy distribution of the simulated pulse. Nevertheless, we find that RR also occupies hyperbola originating at considerably higher frequencies at about $4 \mathrm{PHz}$ than predicted by Eq. (3) with $\rho=0$. In fact, this part of the spectrum can be attributed to blueshifted RR due to the plasma modification of $k(\omega)$. This is shown by the red dashed curve in Fig. 4(b), which represents the hyperbolic component of a solution of Eq. (3) with $\rho=8.3 \times 10^{18} \mathrm{~cm}^{-3}$, the peak electron density in the simulations.

In conclusion, we demonstrated that resonant radiation generation enables relatively long filaments by a new type of collapse-arresting mechanism. Besides the qualitative different self-guiding mechanism, also the properties of the filament are quite distinctive from conventional filamentation. Furthermore, we provided clear evidence that the impact of plasma via its modifying impact on the GVD leads to the excitation of new RR frequencies over wide ranges which are otherwise inaccessible. The whole process not only provides a new concept for light-bullet generation, but also opens up new routes for tailoring light on demand.

We gratefully acknowledge support by the DFG MO 850/20-1, BA 4156/4-1, and Nieders. Vorab ZN3061.

[1] L. Bergé, S. Skupin, R. Nuter, J. Kasparian, and J. P. Wolf, Rep. Prog. Phys. 70, 1633 (2007).

[2] A. Couairon and A. Mysyrowicz, Phys. Rep. 441, 47 (2007).

[3] D. Mihalache, Rom. J. Phys. 59, 295 (2014).

[4] C. Brée, Nonlinear Optics in the Filament Regime, 1st ed. (Springer-Verlag, Berlin, 2012). 
[5] V. P. Kandidov, O. G. Kosareva, I. S. Golubtsov, W. Liu, A. Becker, N. Akozbek, C. M. Bowden, and S. L. Chin, Appl. Phys. B 77, 149 (2003).

[6] C. M. Heyl, H. Coudert-Alteirac, M. Miranda, M. Louisy, K. Kovacs, V. Tosa, E. Balogh, K. Varju, A. L'Huillier, A. Couairon, and C. L. Arnold, Optica 3, 75 (2016).

[7] L. Berge, S. Skupin, C. Köhler, I. Babushkin, and J. Herrmann, Phys. Rev. Lett. 110, 073901 (2013).

[8] F. Silva, D. R. Austin, A. Thai, M. Baudisch, M. Hemmer, D. Faccio, A. Couairon, and J. Biegert, Nat. Commun. 3, 807 (2012).

[9] F. Maucher, T. Pohl, S. Skupin, and W. Krolikowski, Phys. Rev. Lett. 116, 163902 (2016).

[10] D. Faccio, S. Cacciatori, V. Gorini, V. G. Sala, A. Averchi, A. Lotti, M. Kolesik, and J. V. Moloney, Europhys. Lett. 89, 34004 (2010).

[11] J. Kasparian, P. Bejot, J.-P. Wolf, and J. M. Dudley, Opt. Express 17, 12070 (2009).

[12] S. Birkholz, E. T. J. Nibbering, C. Bree, S. Skupin, A. Demircan, G. Genty, and G. Steinmeyer, Phys. Rev. Lett. 111, 243903 (2013).

[13] T. Roger, D. Majus, G. Tamosauskas, P. Panagiotopoulos, M. Kolesik, G. Genty, I. Grazuleviciute, A. Dubietis, and D. Faccio, Phys. Rev. A 90, 033816 (2014).

[14] F. Belgiorno, S. L. Cacciatori, M. Clerici, V. Gorini, G. Ortenzi, L. Rizzi, E. Rubino, V. G. Sala, and D. Faccio, Phys. Rev. Lett. 105, 203901 (2010).

[15] S. Birkholz, C. Bree, A. Demircan, and G. Steinmeyer, Phys. Rev. Lett. 114, 213901 (2015).

[16] P. Panagiotopoulos, P. Whalen, M. Kolesik, and J. V. Moloney, Nat. Photonics 9, 543 (2015).

[17] M. Durand, A. Jarnac, A. Houard, Y. Liu, S. Grabielle, N. Forget, A. Durécu, A. Couairon, and A. Mysyrowicz, Phys. Rev. Lett. 110, 115003 (2013).

[18] Y. Silberberg, Opt. Lett. 15, 1282 (1990).

[19] B. A. Malomed, D. Mihalache, F. Wise, and L. Torner, J. Opt. B 7, R53 (2005).

[20] H. Leblond and D. Mihalache, Phys. Rep. 523, 61 (2013).

[21] B. Malomed, L. Torner, F. Wise, and D. Mihalache, J. Phys. B 49, 170502 (2016).

[22] K. E. Strecker, G. B. Partridge, A. G. Truscott, and R. G. Hulet, Nature (London) 417, 150 (2002).

[23] L. Berge, Phys. Rep. 303, 259 (1998).

[24] D. Majus, G. Tamosauskas, I. Grazuleviciute, N. Garejev, A. Lotti, A. Couairon, D. Faccio, and A. Dubietis, Phys. Rev. Lett. 112, 193901 (2014).

[25] S.-V. Chekalin and A. E. Dokukina and A. E. Dormidonov, V. O. Kompanets, E. O. Smetanina, and V. P. Kandidov, J. Phys. B 48, 094008 (2015).

[26] E. O. Smetanina, V. O. Kompanets, A. E. Dormidonov, S. V. Chekalin, and V. P. Kandidov, Laser Phys. Lett. 10, 105401 (2013).

[27] M. Trippenbach and Y. B. Band, Phys. Rev. A 57, 4791 (1998); S. Skupin and L. Bergé, Physica (Amsterdam) 220D, 14 (2006); J. Liu, R. Li, and Z. Xu, Phys. Rev. A 74, 043801 (2006); L. Bergé and S. Skupin, Phys. Rev. E 71, 065601(R) (2005); M. Hemmer, M. Baudisch, A. Thai, A. Couairon, and J. Biegert, Opt. Express 21, 28095 (2013); B. Shim, S. E. Schrauth, and A. L. Gaeta, Opt. Express 19, 9118 (2011).
[28] M. Durand, K. Lim, V. Jukna, E. McKee, M. Baudelet, A. Houard, M. Richardson, A. Mysyrowicz, and A. Couairon, Phys. Rev. A 87, 043820 (2013).

[29] A. Saliminia, S. L. Chin, and R. Vallée, Opt. Express 13, 5731 (2005).

[30] D. Faccio, A. Averchi, A. Couairon, A. Dubietis, R. Piskarskas, A. Matijosius, F. Bragheri, M. A. Porras, A. Piskarskas, and P. Di Trapani, Phys. Rev. E 74, 047603 (2006).

[31] E. O. Smetanina, V. O. Kompanets, S. V. Chekalin, A. E. Dormidonov, and V. P. Kandidov, Opt. Lett. 38, 16 (2013).

[32] A. E. Dormidonov, V. O. Kompanets, S. V. Chekalin, and V. P. Kandidov, Opt. Express 23, 29202 (2015).

[33] N. Akhmediev and M. Karlsson, Phys. Rev. A 51, 2602 (1995).

[34] A. V. Husakou and J. Herrmann, Phys. Rev. Lett. 87, 203901 (2001).

[35] D. V. Skryabin and A. V. Gorbach, Rev. Mod. Phys. 82, 1287 (2010).

[36] J. M. Dudley and J. R. Taylor, Nat. Photonics 3, 85 (2009).

[37] L. G. Wright, S. Wabnitz, D. N. Christodoulides, and F. W. Wise, Phys. Rev. Lett. 115, 223902 (2015).

[38] I. Babushkin, A. Tajalli, H. Sayinc, U. Morgner, G. Steinmeyer, and A. Demircan, Light Sci. Appl. 6, e16218 (2017).

[39] V. Brasch, M. Geiselmann, T. Herr, G. Lihachev, M. H. P. Pfeiffer, M. L. Gorodetsky, and T. J. Kippenberg, Science 351, 357 (2016).

[40] C. R. Loures, T. Roger, D. Faccio, and F. Biancalana, Phys. Rev. Lett. 118, 043902 (2017).

[41] D. Novoa, M. Cassataro, J. C. Travers, and P. St. J. Russell, Phys. Rev. Lett. 115, 033901 (2015).

[42] Sh. Amiranashvili and A. Demircan, Phys. Rev. A 82, 013812 (2010); Adv. Opt. Technol. 2011, 989515 (2011).

[43] M. V. Ammosov, N. B. Delone, and V. P. Krainov, Sov. Phys. JETP 64, 1191 (1968).

[44] L. Bergé, S. Mauger, and S. Skupin, Phys. Rev. A 81, 013817 (2010).

[45] M. Kretschmar, C. Brée, T. Nagy, A. Demircan, H. G. Kurz, U. Morgner, and M. Kovacev, Opt. Express 22, 22905 (2014).

[46] D. Rayner, A. Naumov, and P. Corkum, Opt. Express 13, 3208 (2005).

[47] J. R. Peñano, P. Sprangle, B. Hafizi, W. Manheimer, and A. Zigler, Phys. Rev. E 72, 036412 (2005).

[48] I. Cristiani, R. Tediosi, L. Tartara, and V. Degiorgio, Opt. Express 12, 124 (2004).

[49] A. Demircan and U. Bandelow, Appl. Phys. B 86, 31 (2007).

[50] R. Driben, A. V. Yulin, and A. Efimov, Opt. Express 23, 19112 (2015).

[51] M. Mlejnek, E. M. Wright, and J. V. Moloney, Opt. Lett. 23, 382 (1998).

[52] S. Linden, H. Giessen, and J. Kuhl, Phys. Status Solidi (b) 206, 119 (1998).

[53] I. Koprinkov, Appl. Phys. B 79, 359 (2004).

[54] See Supplemental Material at http://link.aps.org/ supplemental/10.1103/PhysRevLett.118.163901 for an animated version of Fig. 3(a). 